\title{
Localized Self-Assembly of InAs Nanowire Arrays on Reusable Si Substrates for Substrate-Free Optoelectronics
}

Alireza Abrand ${ }^{1,2}$, Mohadeseh A. Baboli ${ }^{1,2}$, Anastasiia Fedorenko ${ }^{1,2}$, Stephen J. Polly ${ }^{2}$ Evan ManfredaSchulz ${ }^{1,3}$, Seth M. Hubbard ${ }^{1,2,3}$, and Parsian K. Mohseni ${ }^{1,2,4, *}$

${ }^{1}$ Microsystems Engineering, Rochester Institute of Technology, Rochester, NY 14623, USA

${ }^{2}$ NanoPower Research Laboratories, Rochester Institute of Technology, Rochester, NY 14623, USA

${ }^{3}$ School of Physics and Astronomy, Rochester Institute of Technology, Rochester, NY 14623, USA

${ }^{4}$ Department of Electrical and Microelectronic Engineering, Rochester Institute of Technology, Rochester, NY 14623, USA

*To whom correspondences must be addressed: pkmohseni@rit.edu 


\section{Comparison of InAs Localized Self-Assembly and GaAsP Selective-Area Epitaxy on Si}

Figure $\mathrm{S} 1$ shows a comparison of different catalyst-free growth modes for epitaxy of InAs and GaAsP NWs on Si by MOCVD. Figure S1(a) shows a representative SEM image of InAs NWs grown on a bare Si (111) substrate with no masking template. This represents the direct self-assembly growth mode. Resulting NWs show large variances in diameter, length, and spacing. Due to the large lattice mismatch between InAs and Si (i.e., $\sim 11.6 \%$ ), growth proceeds under a pseudo-Volmer-Weber growth regime, which results in randomly-distributed individual NWs. Figure S1(b) show InAs NWs formed under the localized selfassembly (LSA) regime on a Si (111) through an oxide masking template containing a hexagonal array of pores with $500 \mathrm{~nm}$ diameter and $1000 \mathrm{~nm}$ pitch. The growth template serves to define InAs nucleation sites inside the pores while the high lattice mismatch between InAs and Si inhibits lateral expansion of NWs under the growth conditions employed. This results in selective-area self-assembly of NWs that occupy only a fraction of the total pore area. In contrast, Figure $\mathrm{S} 1$ (c) shows $\mathrm{GaAs}_{0.73} \mathrm{P}_{0.27}$ nanocrystals grown under the selective-area epitaxy regime on a Si (111) substrate containing the same oxide masking template as that used for InAs LSA growths. Nucleation is limited to the pore sites. Due to the smaller lattice mismatch between $\mathrm{GaAs}_{0.73} \mathrm{P}_{0.27}$ and $\mathrm{Si}$ (i.e., $\sim 3.1 \%$ ), the nanocrystals laterally extend to fully occupy all pores. Thus, the LSA growth mode exhibits a combination of the characteristics of the direct self-assembly and selectivearea epitaxy regimes.
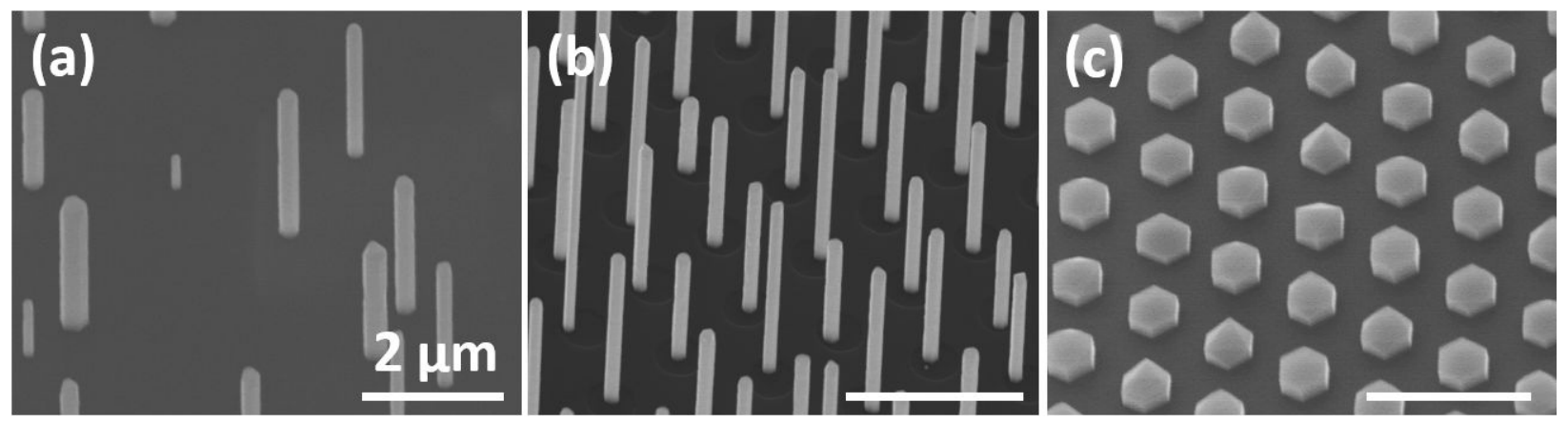

Figure S1. Tilted-view SEM images of (a) direct self-assembly growth of InAs on bare Si (111) with no template, (b) localized self-assembly growth of InAs on Si substrate with masking template of $500 \mathrm{~nm}$ diameter pores with $1000 \mathrm{~nm}$ pitch, and (c) selective-area epitaxy of GaAsP on Si using the same masking template as shown in (b) and under otherwise similar growth conditions. 


\section{InAs Nanowire Tip Analysis}

To better understand whether LSA growth of InAs NWs proceeds via the In droplet-assisted mechansim or the self-assembly mechanism, the tip structure of NWs is investigated. Since a potential In droplet at the NW tip will crystalize as InAs during the post-growth cooling stage under a continual supply of $\mathrm{AsH}_{3}$, an additional growth is carried out under Sample C conditions, but growth is terminated by simultaneously stopping the supply of both TMIn and $\mathrm{AsH}_{3}$ precursors. Any In droplets responsible for NW growth should be observable in such a sample, as has previously been observed by Gao and colleagues [S1]. After growth, NWs are removed from the growth substrate via sonication and deposited on lacey carbon TEM grids. Similar to previously published work [S2], the tip segments of the deposited NWs can be identified under TEM based on a either truncated facet profile in the case of seed-free self-assembly growth or based on the presence of a separate In phase in the case of VLS growth. Figure S2 shows (a) a schematic representation of the growth sequence with temperature and precursor flows over time; (b) TEM image of an InAs NW grown under the modified Sample $\mathrm{C}$ conditions with $\mathrm{AsH}_{3}$-free cooling stage; and (c) EDXS linescan showing X-ray signal intensities representative of elemental In (black) and As (red) as measured over the positions indicated by the white dotted line in (b). In Figure S2(b), no separate In droplet is observed at the NW tip. The EDXS linescans show no noticeable variation in the relative In and As signal intensities along
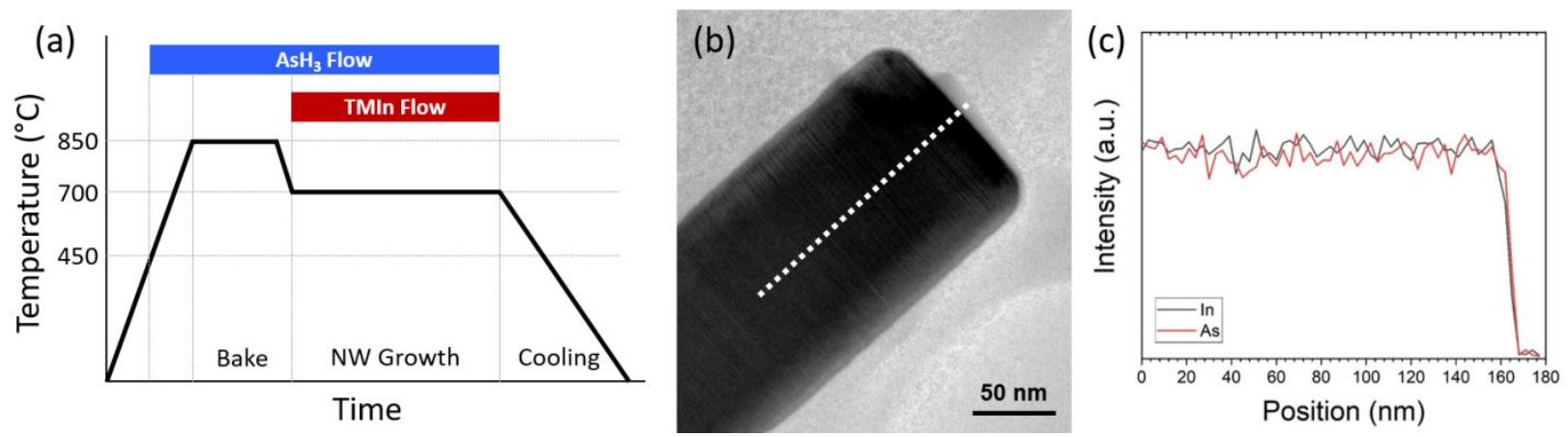

Figure S2. (a) Growth sequence employed for InAs NW growth with simultaneous termination of $A_{s} H_{3}$ and TMIn precursors. (b) TEM image of the tip of an InAs NW grown under the sequence depicted in (a). (c) EDXS linescan corresponding to location marked by a white dotted line in (b). Elemental counts of In and As are shown in black and red, respectively. 
the tip of the NW. This indicates that the tip region is composed of a single InAs phase. We believe the absence of a separate In phase at the NW tip demonstrates the likelihood that growth of InAs NWs in the current work does not proceed via the VLS mode. 


\section{Piranha Surface Treatment for Substrate and Masking Template Restoration}

Two approaches for Si substrate restoration after NW array delamination are explored. In the first approach, the post-delamination growth substrates are subjected to a citric acid $\left(\mathrm{C}_{6} \mathrm{H}_{8} \mathrm{O}_{7}: \mathrm{H}_{2} \mathrm{O}_{2}, 20: 1\right)$ treatment for 5 minutes to selectively etch the remnant InAs NW base segments inside the template nanopores. In the second approach, the growth substrates are treated by piranha solution $\left(\mathrm{C}_{2} \mathrm{SO}_{4}: \mathrm{H}_{2} \mathrm{O}_{2}, 3: 1\right)$ for 5 minutes to return the oxide-templated parent substrate to its pre-growth condition. Figure S3(a) shows the postdelamination parent substrates with oxide masking layers. Figures S3(b) and (c) show images of the substrate and masking template after citric acid and piranha solution treatments, respectively. In Figure S3(a), the remnant InAs NW base segments are observed inside the template pores. In both cases, no InAs crystallites are observed after the selective etching treatments and the masking template is preserved for reuse. The citric acid treatment is favored over the piranha solution treatment in this work due to chemical compatibility with subsequent device fabrication processes; namely, for the height adjustment process to chemically etch extended InAs NW tip segments.
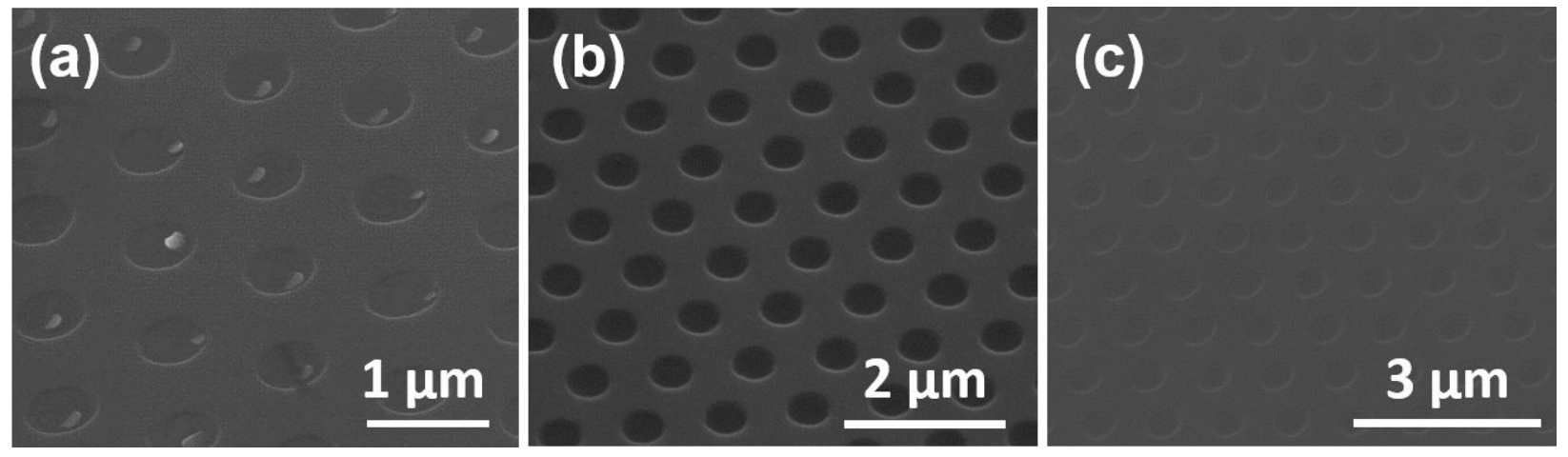

Figure S3. Tilted-view SEM images of (a) parent Si substrate after InAs NW growth and delamination, (b) after citric acid treatment, and (c) after piranha solution treatment. 


\section{Analytical Transmission Electron Microscopy of InAs NW Growth on Reused Si Substrate}

Figure S4(a) shows a high-resolution transmission electron microscopy (HR-TEM) image obtained along the InAs/Si interface. The InAs lattice extends along the vertical growth direction and stems from the remnant base of a NW on the parent Si substrate, allowing substrate reuse without restoration. Figure S4(b) shows a selected-area electron diffraction (SAED) pattern collected at the InAs/Si interface along the $\langle 1 \overline{1} 0\rangle$ zone axis. Three sets of distinguishable diffraction spots are indexed. These include reflections from the $\mathrm{Si}$ substrate (white) as well as a combination of diffraction spots stemming from zincblende InAs (yellow) and wurtzite InAs (blue) segments. The superposition of SAED patterns characteristic of both cubic and hexagonal InAs phases and visible streaking along the $\langle\overline{1} \overline{1} \overline{1}\rangle$ direction highlight the mixed-phase crystal structure of NWs grown under the selective-area self-assembly mode. Figures S4(c) and (d) show fast Fourier transform (FFT) patterns generated from HR-TEM images collected at the InAs/Si interface (i.e., representative of the InAs NW remnant base segment) and along the NW body (i.e., representative of the “regrown” InAs lattice), respectively. The matching FFT pattern symmetry and consistent streaking along the $[\overline{1} \overline{1}]$ direction indicates that the mixed phase crystal structure is common to both the originally grown and the regrown NW segments. Figure S4(e)-(h) show a high-angle annular dark-field scanning transmission electron microscopy (HAADF-STEM) image and elemental In, As, and Si maps with superimposed linescans, respectively, from the corresponding area collected using energy-dispersive X-ray spectrometry (EDXS). The yellow line indicates the EDXS linescan location, corresponding to the NW axial direction. The slight reduction in elemental In and As linescan intensity near the top of the NW is due to the reduced thickness of the wedge-shaped TEM lamella. A uniform InAs NW composition is confirmed in Figure S4(f) and (g). No lattice discontinuities are observed along the anticipated regrowth plane location in (a), nor are changes in composition observed near the base of the NW in (f) and (g). A sharp contrast change below the NW basal plane in (e) and corresponding rapid increase in elemental Si counts in (f) are indicative of an abrupt InAs/Si interface. 

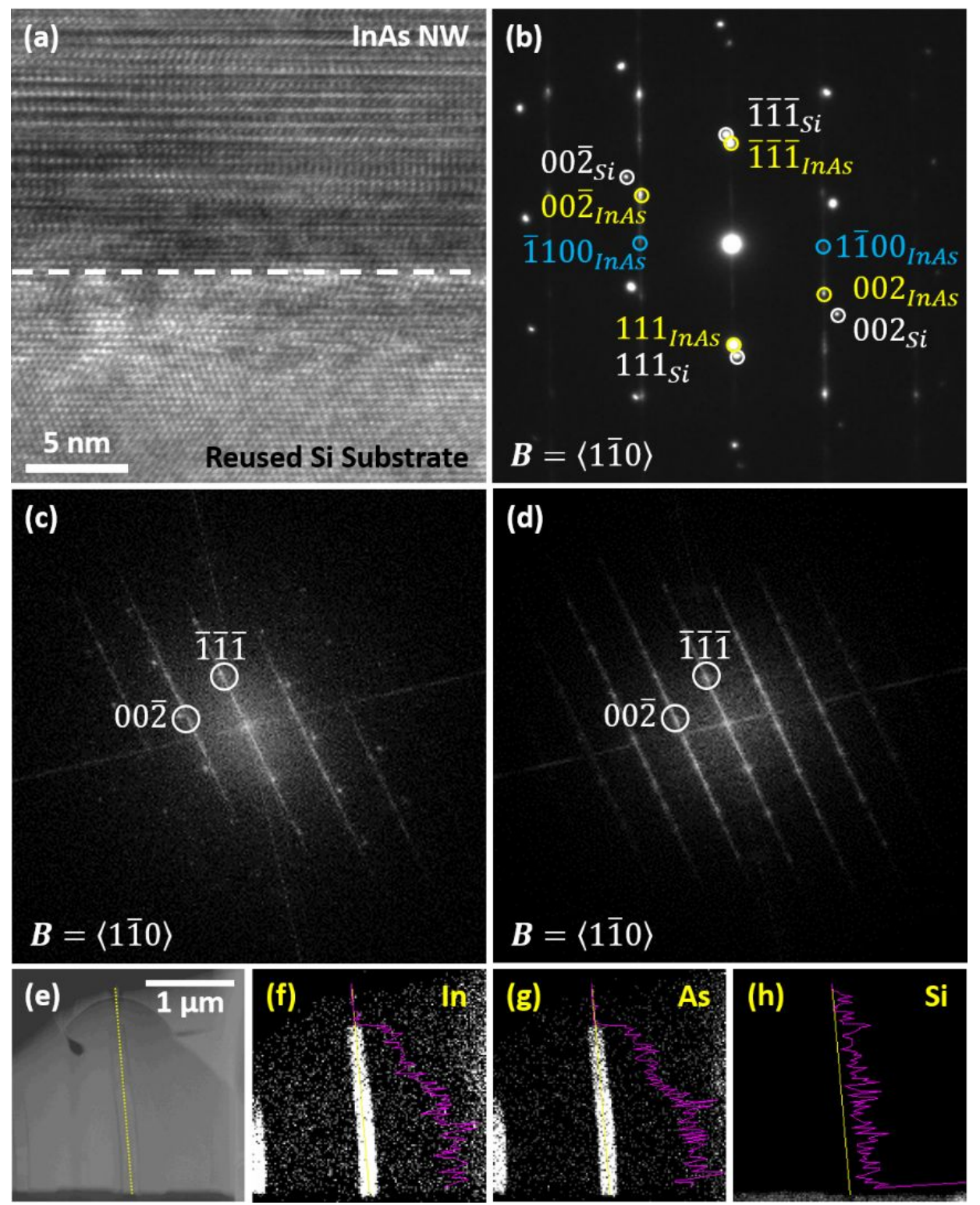

Figure S4. (a) HR-TEM image and (b) SAED pattern collected along the interface of a regrown InAs NW and parent Si substrate. FFT patterns generated from HR-TEM images collected at (c) the InAs/Si interface and (d) near the longitudinal midpoint of the regrown $N W$. (e) HAADF-STEM image and (f)-( $h)$ corresponding area EDXS maps with superimposed linescans representing counts of elemental In, As, and Si, respectively. 


\section{Statistical Analysis of Alignment Angle of Delaminated Nanowires}

Preserving the as-grown orientation of NWs after delamination and transfer to carrier wafers is necessary for processing simplicity and for reliable and reproducible performance of NW array-based membrane devices. Here, the Colorless First Contact ${ }^{\mathrm{TM}}(\mathrm{CFC})$ polymer is used as a delamination matrix that introduces minimal damage to the NWs and enables their as-grown separation and vertical orientation to be maintained upon peel-off and transfer to foreign carrier wafers. Figure S5(a) shows a representative distribution of the alignment angle of $100 \mathrm{NWs}$ measured from an as-grown sample. A mean orientation angle of $89.95^{\circ}$ is measured with a standard deviation value of $0.35^{\circ}$. Figure S5(b) shows the alignment angle distribution of 100 NWs measured after array transfer to a carrier wafer and CFC polymer dissolution. The free-standing and transferred NWs are anchored at their bases by the trilayer backside metallic contact stack. A mean orientation angle of $89.94^{\circ}$ and standard deviation of $0.69^{\circ}$ are measured after transfer. In the case of the transferred array, approximately $90 \%$ of all NWs are oriented between $89^{\circ}$ to $91^{\circ}$. The remaining $10 \%$ of NWs are aligned between $88^{\circ}-89^{\circ}$ and $91^{\circ}-92^{\circ}$. No NWs are observed with orientation angles less than $88^{\circ}$ or greater than $92^{\circ}$, demonstrating that their verticality is largely preserved upon peel-off, transfer, and dissolution of the delamination polymer.
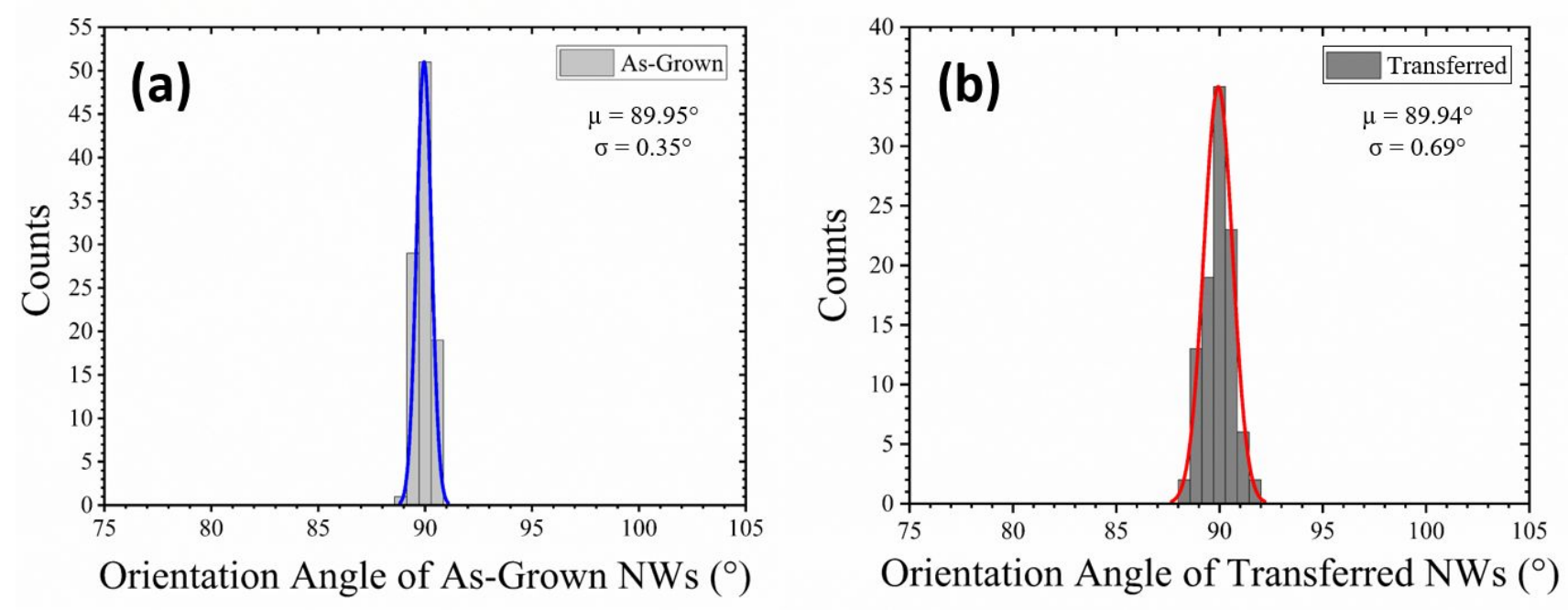

Figure S5. Histogram of orientation angle of (a) as-grown and (b) transferred NWs. The blue curve in (a) and the red curve in (b) show data fit to a Gaussian distribution. 


\section{References}

[S1] Gao, Q.; Dubrovskii, V. G.; Caroff, P.; Wong-Leung, J.; Li, L.; Guo, Y.; Fu, L.; Tan, H. H.; Jagadish, C. Simultaneous Selective-Area and Vapor-Liquid-Solid Growth of InP Nanowire Arrays. Nano Lett. 2016, 16 (7), 4361-4367.

[S2] Mohseni, P. K.; Behnam, A.; Wood, J. D.; English, C.; Lyding, J. W.; Pop, E.; Li, X. $\operatorname{In}_{x}$ Ga $_{1-x} A s$ Nanowire Growth on Graphene: van der Waals Epitaxy Induced Phase Segregation. Nano Lett. 2013, 13 (3), 1153-1161. 